$\Rightarrow$ SYNAPTIC PLASTICITY

\section{Polymerize and learn?}

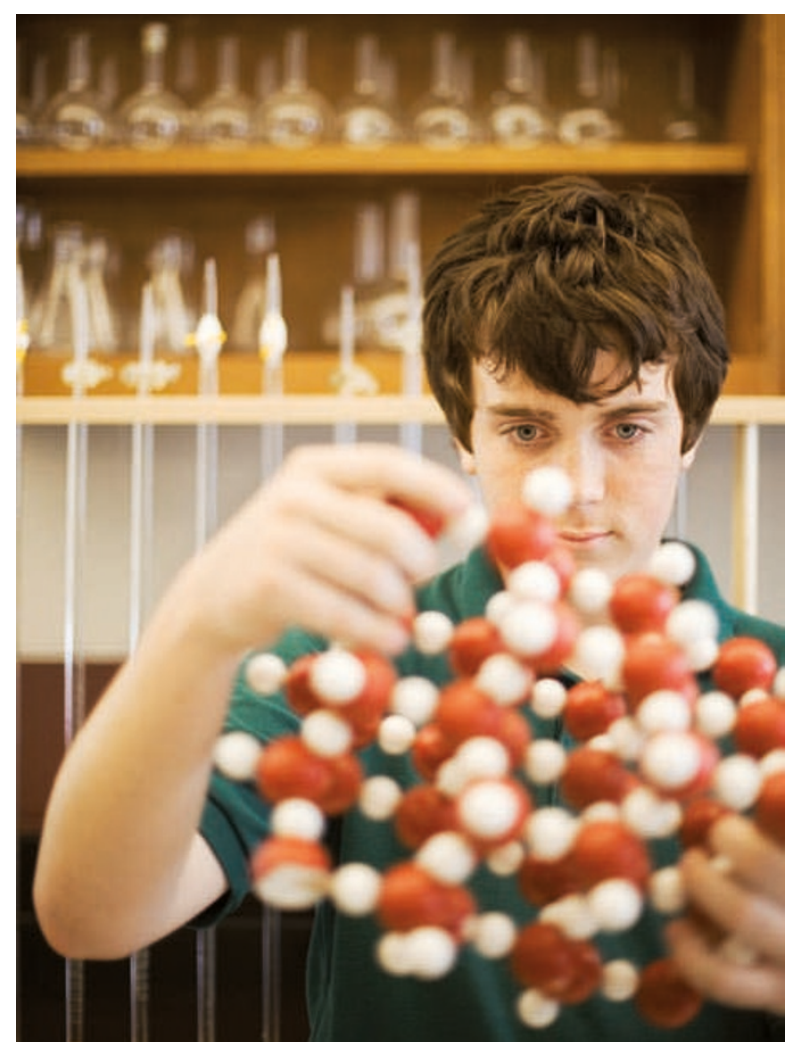

Activity-dependent changes at the synapse require protein synthesis in order to persist for extended periods and to contribute to the formation of long-term memories. However, the proteins involved in the stabilization of synaptic changes are largely unknown. Now, Si et al. show that in Aplysia spp. a prion-like protein that transitions to a multimeric state in response to pulses of serotonin may be a key mediator of these long-term changes in the synapse.

A neuron-specific form of the cytoplasmic polyadenylation element binding protein (ApCPEB) has an amino-terminal prion-like domain and had previously been shown to be required for synaptic facilitation in Aplysia spp. - a phenomenon analogous to long-term potentiation. When the authors overexpressed ApCPEB in sensory neurons, it formed punctate structures that were amyloid in nature, which is a common characteristic of all prions tested so far. To directly investigate whether these puncta were the product of protein multimerization, the authors used a fluorescence reconstitution assay in which two halves of a fluorescence protein were attached to ApCPEB monomers. As expected for proteins that physically interact to form homotypic multimers, punctate fluorescence was observed in sensory neurons expressing the constructs. Furthermore, when the authors attached ApCPEB to a green fluorescent protein that can be irreversibly converted to red after a brief light excitation, the proportion of green fluorescence increased gradually after the multimers had been entirely converted to red, suggesting that they continuously recruited new proteins.

The conformational change between the monomeric and multimeric state is thought to occur spontaneously in prion-like proteins.
Surprisingly, when the authors stimulated ApCPEB-expressing neurons in vitro with pulses of serotonin, the number of detectable puncta increased, suggesting that multimerization of ApCPEB is directly regulated by synaptic activity. Moreover, injecting an antibody that preferentially binds the multimeric form of ApCPEB into sensory neurons in a sensorymotor neuron culture abolished synaptic facilitation 48 hours after stimulation, whereas basal synaptic transmission and facilitation after 24 hours remained unaffected. This result indicates that ApCPEB multimers are specifically involved in the long-term stabilization of changes in synaptic efficacy.

Prion-like proteins are known to convert spontaneously into a multimeric form, generating insoluble amyloids that cause prion-associated pathologies. However, ApCPEB seems to be unique in that, despite its prion-like features, even in a multimeric state it retains its biochemical activity and therefore its potential for physiological function. Moreover, this conversion is dependent on a neurotransmitter signal, making it an unexpected but particularly appropriate candidate for signal transduction. Future studies should clarify the cellular mechanisms by which serotonin regulates this conversion and whether this novel role for prion multimers in Aplysia spp. can be extended to other species.

Cristian Bodo

ORIGINAL RESEARCH PAPER $\mathrm{Si}$, K. et al.

Aplysia CPEB can form prion-like multimers in sensory neurons that contribute to long-term facilitation. Cell 140, 421-435 (2010) 